\title{
Circulating Current Injection Methods Based on Instantaneous Information for the Modular Multilevel Converter
}

\author{
Josep Pou, Senior Member, IEEE, Salvador Ceballos, Georgios Konstantinou, Member, IEEE, \\ Vassilios G. Agelidis, Senior Member, IEEE, Ricard Picas, Student Member, IEEE, \\ and Jordi Zaragoza, Member, IEEE
}

\begin{abstract}
This paper studies different circulating current references for the modular multilevel converter (MMC). The circulating current references are obtained from the instantaneous values of the output current and modulation signal of the phase-leg. Therefore, determination of the amplitude and phase of the output current is not needed, which is a significant improvement compared to other methods such as those based on injecting specific harmonics in the circulating currents. Among the different methods studied in this paper, a new method is introduced, which is able to reduce the capacitor voltage ripples compared to the other methods. A closed-loop control is also proposed which is able to track the circulating current references. With the discussed methods the average values of the capacitor voltages are maintained at their reference while the voltage ripples are kept low. Experimental results are presented to demonstrate the effectiveness of the proposed and discussed methods.
\end{abstract}

Index Terms-Modular multilevel converter, Circulating current control, Arm current, Capacitor voltage ripple.

\section{INTRODUCTION}

$\mathbf{M}$ ULTILEVEL converters have attracted significant interest for medium/high power applications. Among various multilevel converter topologies [1], the modular multilevel converter (MMC) [2]-[6], offers several salient features which make it a potential candidate for various applications including high-voltage direct current (HVDC) transmission systems [7][9], flexible alternating current transmission system (FACTS) controllers [10], photovoltaic generation [11], wind turbine applications [12], and motor drives [13]-[15]. The most attractive features of an MMC are (i) its modularity and scalability to

This work has been supported by the Ministerio de Economía y Competitividad of Spain under project ENE2012-36871-C02-00.

J. Pou is with the Australian Energy Research Institute and the School of Electrical Engineering and Telecommunications, The University of New South Wales, Sydney, NSW, 2052, Australia, on leave from the Technical University of Catalonia, Barcelona, 08034, Catalonia, Spain (e-mail: j.pou@ unsw.edu.au, josep.pou@upc.edu).

S. Ceballos is with the Energy Unit, Tecnalia Research and Innovation, Derio, 48160, Basque Country, Spain (e-mail: salvador.ceballos@tecnalia.com).

G. Konstantinou and V. G. Agelidis are with the Australian Energy Research Institute and the School of Electrical Engineering and Telecommunications, The University of New South Wales, Sydney, NSW, 2052, Australia (e-mail g.konstantinou@unsw.edu.au, vassilios. agelidis@unsw.edu.au).

R. Picas and J. Zaragoza are with the Terrassa Industrial Electronics Group, Technical University of Catalonia, Terrassa, 08222, Catalonia, Spain (e-mail ricard.picas@upc.edu, jordi.zaragoza-bertomeu@upc.edu). different power and voltage levels, and (ii) its relatively simple capacitor voltage balancing task [9].

Capacitor voltage balancing of an MMC does not have the limitations and complexities associated with other multilevel converters [16], [17]. However, it is mutually coupled with the circulating currents within each phase-leg of the MMC. Analysis of the circulating currents of an MMC has been reported in the technical literature [18]-[20]. Improper control of the circulating currents can have adverse impacts on the ratings of the MMC components and power losses. In [21], a closed-loop control strategy based on eliminating the second order harmonic of the arm currents was proposed. Similarly in [22], two control strategies were introduced to eliminate the ac components in the circulating current. Although these strategies reduce the rms value of the arm currents and therefore the power losses of the MMC, the capacitor voltage ripples can be reduced further. It should be remarked that reducing the capacitor voltage ripples is an important target because it enables the use of smaller capacitors [23]. This eventually helps reduce the cost of the MMC due to the large number of capacitors integrated in the topology. In [24], a second harmonic is injected into the circulating currents of the MMC to achieve a reduction in the capacitor ripples. The injection of a fourth harmonic in addition to the second one is also considered in [25], [26]. The main drawback of these methods is that they rely on the determination of the amplitude and phase of the output currents of the MMC and the use of an extensive look-up table. Determination of proper references for the circulating currents obtained from instantaneous magnitudes is shown in [27], [28]. These methods only require the use of the instantaneous values of the output current and the reference signal of the phase-leg for the determination of the circulating current. Although minimum capacitor voltage ripples are not achieved, the results obtained are very close to the optimal ones.

The inclusion of a higher frequency circulating current component in coordination with a zero-sequence injection to the reference signals helps attenuate the low frequency ripples in the capacitor voltages [13]-[15]. However, this high frequency zero-sequence signal may produce a zero-sequence current depending on the application and grounding connection of the MMC. Additionally, in a grid-connected converter operating at $50 \mathrm{~Hz}$ or $60 \mathrm{~Hz}$, the injected component has to be at a relatively higher frequency (above $1 \mathrm{kHz}$ ). Injection of 
larger arm voltages for the control of the circulating currents due to the higher impedance of the arm inductors at this frequency is required. This is why the method in [13]-[15] is especially interesting for motor drive applications when the motor operates at a low speed/frequency, and it not convenient for grid-connected applications.

In this paper, circulating current references based on instantaneous information of the converter are studied. The references are derived from a comprehensive analysis of the MMC based on evaluating the oscillating energy/power in the arms. Three references are obtained for the circulating current, the first one injects only a dc component, which is based on [21], [22]. The second one was already presented in [28], and it is same one than that introduced in [27], although it was derived from a different approach. The third circulating current reference is a new approach introduced in this paper. Based on the mathematical model of an MMC developed in [18], the normalized capacitor voltage ripple amplitudes are evaluated for all operating conditions of the MMC. This information can be used to size the capacitors of the converter for different applications and operating conditions. Furthermore, a closedloop control scheme for the circulating currents of the MMC is presented. The effectiveness of the different methods in terms of reducing the amplitude of the capacitor voltage ripples is evaluated in an laboratory prototype with five SMs per arm.

The rest of this paper is organized as follows. Section II presents the common and differential circuits of an MMC phase-leg. In Section III, the power in the upper and lower arms is calculated and used in Section IV to define circulating current references for the MMC. In Section V the inclusion of a zero sequence into the reference signals is considered. SM capacitor voltage ripple amplitudes and rms arm currents are presented and compared in Section VI according to the different circulating current references. In Section VII, the results obtained from the circulating current references studied in this paper are compared and benchmarked against those in [26]. In Section VIII a circulating current control is introduced. Section IX reports the experimental results, and Section $\mathrm{X}$ concludes this paper.

\section{COMmon And DifFerential Mode CiRcuits}

Fig. 1(a) shows a general circuit representation of an MMC phase-leg. A three-phase MMC consists of six arms where each arm includes $N$ series-connected, identical, halfbridge sub-modules (SMs). Although other SM configurations have been considered in the literature [29], [30], the most extensively used is the half-bridge topology because of its simplicity. Reactors $L$ within the converter arms offer control of the circulating currents and limit the fault currents. The output voltage of each $\mathrm{SM}\left(v_{S M}\right)$ is either equal to its capacitor voltage $\left(v_{C}\right)$ or zero, depending on the switching states of the switch pair $s_{1}$ and $s_{2}$ in each SM.

Fig. 1(b) represents a phase-leg showing only the activated SMs in the arms. Since the output voltage of the non-activated SMs is zero, those SMs do not insert their capacitors in the arms and they are not included in Fig. 1(b). The common and differential voltages applied to the extremes of the inductors

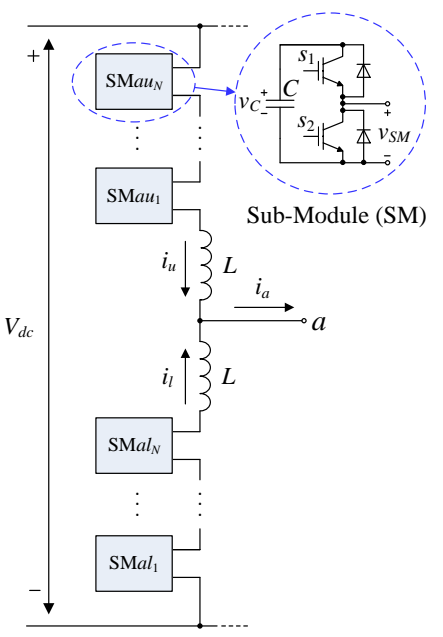

(a)

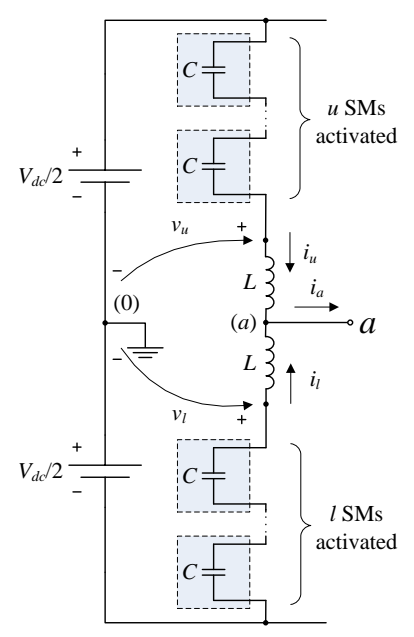

(b)
Fig. 1. MMC phase-leg: (a) Circuit diagram and (b) circuit with activated SMs.

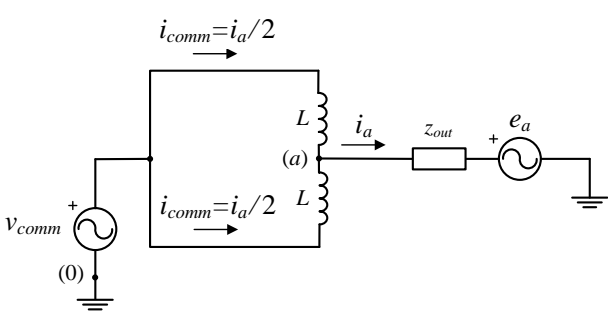

(a)

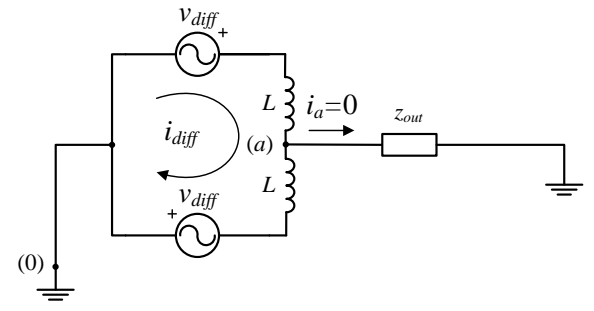

(b)

Fig. 2. Equivalent phase-leg: (a) common and (b) differential mode circuits.

are:

$$
\begin{gathered}
v_{c o m m}=\frac{v_{u}+v_{l}}{2} \text { and } \\
v_{\text {diff }}=\frac{v_{u}-v_{l}}{2} .
\end{gathered}
$$

Assuming that the phase-leg is connected to a grid voltage $e_{a}$ through an impedance $z_{\text {out }}$ and applying the principle of superposition, common and differential mode circuits can be obtained, as shown in Fig. 2(a) and Fig. 2(b), respectively. The common and differential arm currents are:

$$
\begin{gathered}
i_{c o m m}=\frac{i_{u}+i_{l}}{2}=\frac{i_{a}}{2} \text { and } \\
i_{\text {diff }}=\frac{i_{u}-i_{l}}{2} .
\end{gathered}
$$

From (3) and (4) the arm currents can be deduced as follows:

$$
i_{u}=i_{c o m m}+i_{d i f f}=\frac{i_{a}}{2}+i_{d i f f} \text { and }
$$




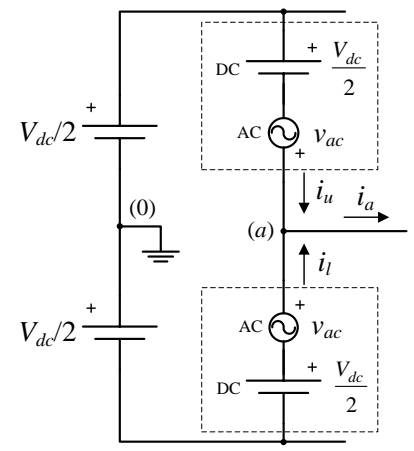

Fig. 3. Scheme of the phase-leg with equivalent voltages.

$$
i_{l}=i_{\text {comm }}-i_{\text {diff }}=\frac{i_{a}}{2}-i_{d i f f} .
$$

The two circuits in Fig. 2(a) and (b) can be analyzed independently of each other. The differential voltage can be used to control the differential current within the phase-leg, without producing any distortion to the output current.

According to Fig. 2(b), the differential current will be:

$$
i_{\text {diff }}=\frac{1}{L} \int_{0}^{t} v_{\text {diff }} d t+I_{\text {diff } 0},
$$

where $I_{\text {diffo } 0}$ is the initial value of the differential current.

In this paper, the differential current is also referred to as circulating current. This is because it circulates through the arms of the phase-leg without appearing in the output current $\left(i_{a}\right)$. It is demonstrated in Section III that the differential current has to contain a dc component $\left(I_{d c}\right)$ that is essential to keep the arms energized; i.e. maintain the capacitor voltages around their reference value. On the other hand, some ac current components $\left(i_{d i f f_{a c}}\right)$ can be defined to meet certain objectives, such as minimizing the voltage ripples in the SM capacitors or the rms value of the arm currents to improve the MMC efficiency. Therefore, generally speaking the differential current will be composed by the following two terms:

$$
i_{d i f f}=I_{d c}+i_{d i f f_{a c}} .
$$

\section{InStANTANEOUS POWER IN THE ARMS}

The SM capacitor voltages are closely connected to the energy in the arms. Therefore, it is necessary to analyze the energy variation in the arms, which is related to the power in the arms, in order to develop a proper control for the MMC. Fig. 3 represents the steady state voltages generated within the arms of the MMC. It is assumed that the arm inductors are small and consequently the voltage drop and the energy in the inductors are both small. Under this assumption, the power in the arms will be:

$$
\begin{gathered}
p_{u}=\left(\frac{V_{d c}}{2}-v_{a c}\right) i_{u} \text { and } \\
p_{l}=-\left(\frac{V_{d c}}{2}+v_{a c}\right) i_{l} .
\end{gathered}
$$

The ac component of the reference voltage of the arm $\left(v_{a c}\right)$, which ranges in the interval $\left[-V_{d c} / 2,+V_{d c} / 2\right]$, and the output current of the MMC are assumed to be sinusoidal:

$$
v_{a c}=v_{a m} \frac{V_{d c}}{2}=m_{a} \frac{V_{d c}}{2} \cos (\omega t) \text { and }
$$

$$
i_{a}=\hat{I}_{a} \cos (\omega t+\varphi),
$$

where $v_{a m}$ represents the modulation or reference signal normalized within the range $[-1,1]$, and $m_{a}$ the modulation index. Substituting (5), (6), (11) and (12) into (9) and (10), the power in the upper and lower arms become:

$$
\begin{array}{r}
p_{u}=-\frac{m_{a} V_{d c} \hat{I}_{a}}{8} \cos (\varphi)+\frac{V_{d c} \hat{I}_{a}}{4} \cos (\omega t+\varphi)- \\
\frac{m_{a} V_{d c} \hat{I}_{a}}{8} \cos (2 \omega t+\varphi)+\frac{V_{d c}}{2} i_{d i f f}-\frac{m_{a} V_{d c}}{2} \cos (\omega t) i_{d i f f}
\end{array}
$$

$$
\begin{array}{r}
p_{l}=-\frac{m_{a} V_{d c} \hat{I_{a}}}{8} \cos (\varphi)-\frac{V_{d c} \hat{I_{a}}}{4} \cos (\omega t+\varphi)- \\
\frac{m_{a} V_{d c} \hat{I_{a}}}{8} \cos (2 \omega t+\varphi)+\frac{V_{d c}}{2} i_{d i f f}+\frac{m_{a} V_{d c}}{2} \cos (\omega t) i_{d i f f} .
\end{array}
$$

In steady state, no dc power component should appear in the arms, otherwise the accumulated energy in the capacitors will increase or decrease continuously. Therefore, the differential current $i_{\text {diff }}$ has to contain a dc component able to compensate for the first term in (13) and (14). The other terms show that there will be power oscillations in the arms and therefore voltage ripples in the capacitors. These voltage ripples can be reduced by implementing a proper differential current control.

\section{Differential CURREnt REFERENCES}

\section{A. A dc Differential Current}

In order to reduce the power losses in the semiconductors of the MMC, the rms values of the arm currents should be minimized. This can be achieved by imposing a differential current that contains a dc component only $\left(i_{d i f f_{a c}}=0\right)$ :

$$
i_{d i f f}=I_{d c} .
$$

Substituting (15) into (13) and (14), and forcing the dc power term to be zero, one can deduce that:

$$
i_{d i f f}=I_{d c}=\frac{m_{a} \hat{I}_{a}}{4} \cos (\varphi) .
$$

Under this assumption, the powers in the arms become:

$$
\begin{gathered}
p_{u}=\frac{V_{d c} \hat{I_{a}}}{4} \cos (\omega t+\varphi)-\frac{m_{a}^{2} V_{d c} \hat{I_{a}}}{8} \cos (\varphi) \cos (\omega t) \\
-\frac{m_{a} V_{d c} \hat{I_{a}}}{8} \cos (2 \omega t+\varphi) \text { and } \\
p_{l}=-\frac{V_{d c} \hat{I_{a}}}{4} \cos (\omega t+\varphi)+\frac{m_{a}^{2} V_{d c} \hat{I_{a}}}{8} \cos (\varphi) \cos (\omega t) \\
\quad-\frac{m_{a} V_{d c} \hat{I_{a}}}{8} \cos (2 \omega t+\varphi) .
\end{gathered}
$$

Equations (17) and (18) show that the power and energy in the arms will oscillate with angular frequencies $\omega$ and $2 \omega$. Oscillations of power/energy in the overall phase-leg are given by adding $p_{u}$ and $p_{l}$. In this case, $p_{u}+p_{l}$ only includes a second harmonic term.

The rms value of the arm currents is:

$$
I_{u_{r m s}}=I_{l_{r m s}}=\frac{\hat{I}_{a}}{2 \sqrt{2}} \sqrt{\frac{m_{a}^{2}}{2} \cos ^{2}(\varphi)+1},
$$

which is the minimum value achievable and hence maximum efficiency of the MMC can be achieved under such operating conditions. 


\section{B. Injection of a Second Order Harmonic}

In addition to the dc current component, a second harmonic can be injected into the differential current, as follows:

$$
i_{d i f f}=I_{d c}+\hat{I_{2}} \cos \left(2 \omega t+\varphi_{2}\right) .
$$

Substituting (20) into the power equations (13) and (14), one can conclude that the second order power oscillations can be canceled by injecting the following differential current:

$$
i_{d i f f}=\frac{m_{a} \hat{I}_{a}}{4} \cos (\varphi)+\frac{m_{a} \hat{I}_{a}}{4} \cos (2 \omega t+\varphi) .
$$

Then, the arm powers become:

$$
\begin{gathered}
p_{u}=\frac{V_{d c} \hat{I_{a}}}{4} \sqrt{1+\frac{m_{a}^{2}}{2}\left[\frac{m_{a}^{2}}{8}-1+\left(m_{a}^{2}-2\right) \cos ^{2} \varphi\right]} \\
\cos \left(\omega t+\varphi^{\prime}\right)-\frac{m_{a}^{2} V_{d c} \hat{I}_{a}}{16} \cos (3 \omega t+\varphi) \text { and } \\
p_{l}=-p_{u}, \\
\varphi^{\prime}=\operatorname{atan} \frac{\left(4-m_{a}^{2}\right) \sin \varphi}{\left(4-3 m_{a}^{2}\right) \cos \varphi} .
\end{gathered}
$$

It can be observed that the sum of $p_{u}$ and $p_{l}$ is zero in this case; this means that there is no power and energy oscillations within the whole phase-leg. The second order power oscillations that appear in (17) and (18) are canceled in (22) and (23). A third harmonic appears instead; however, it has smaller amplitude. Additionally, the first-order power oscillation term $(\omega)$ has lower amplitude than in the previous case. These factors lead to a reduction in the capacitor voltage ripple amplitudes when compared to the case of injecting only a dc differential current and the rms value of the arm currents would be:

$$
I_{u_{r m s}}=I_{l_{r m s}}=\frac{\hat{I}_{a}}{2 \sqrt{2}} \sqrt{\frac{m_{a}^{2}}{2}\left[1+\cos ^{2}(\varphi)\right]+1} .
$$

The addition of the second order harmonic into the differential current increases the rms value of the arm currents compared to (19) and therefore additional power losses will be produced. Nevertheless, a proper second harmonic in the arm currents will reduce the capacitor voltage ripples. However, according to (21), the amplitude and phase of the output current need to be determined in order to define the proper second harmonic for the differential current.

\section{Determination of the Differential Current from Instanta- neous Values. Method 1}

In this subsection, the reference of the differential current is determined from the instantaneous value of the output current of the phase-leg. The target is the same as in the previous case, i.e. the injection of the proper value of the dc current and the second order harmonic given by (21).

In order to produce low ripples to the capacitor voltages, the arm that inserts fewer capacitors connected in series (i.e. higher equivalent capacitance) should carry more output current. This would happen naturally if the inductors $L$ in the model in Fig. 1(b) were assumed to be zero [18]. Although it is a simplified representation of the system, the currents produced in the arms under this assumption will help define a proper differential current reference.

Let us define the upper and lower phase-arm capacitors $C_{u}$ and $C_{l}$ as the instantaneous values of the total capacitances that are inserted by the activated SMs in the upper and lower arms. Consequently, the number of activated SMs at any instant defines the value of $C_{u}$ and $C_{l}$. If the variables $u$ and $l$ are the number of series connected SMs in the upper and lower arms, respectively, the instantaneous values of the arm capacitors are:

$$
\begin{gathered}
C_{u}=\frac{C}{u} \text { and } \\
C_{l}=\frac{C}{l} .
\end{gathered}
$$

The phase current $i_{a}$ is shared between the upper and the lower phase-arms based on:

$$
\begin{gathered}
i_{u}=i_{a} \frac{C_{u}}{C_{u}+C_{l}}=i_{a} \frac{l}{u+l} \text { and } \\
i_{l}=i_{a} \frac{C_{l}}{C_{u}+C_{l}}=i_{a} \frac{u}{u+l} .
\end{gathered}
$$

The locally averaged value of $u$ and $l$ calculated over a switching period can be represented by:

$$
\begin{gathered}
u=N \frac{1-v_{a m}}{2} \text { and } \\
l=N \frac{1+v_{a m}}{2} .
\end{gathered}
$$

For the sake of simplicity, no change in the notation of $u$ and $l$ when dealing with locally-averaged magnitudes is made. Substituting (30) and (31) into (28) and (29), the arm currents become:

$$
\begin{gathered}
i_{u}=i_{a} \frac{1+v_{a m}}{2} \text { and } \\
i_{l}=i_{a} \frac{1-v_{a m}}{2} .
\end{gathered}
$$

Equations (32) and (33) provide the instantaneous references for the arm currents. Considering (4), the differential term is:

$$
i_{d i f f}=\frac{i_{a} v_{a m}}{2} .
$$

Equation (34) provides the differential current reference obtained directly from the instantaneous values of the output current and the modulation signal. If the reference signal and the output current are sinusoidal, as it was assumed in (11) and (12), respectively, the differential current becomes identical to (21). Note, however, that in this case the current reference is obtained from the instantaneous values given in (34) and there is no need to determine the amplitude and phase of the output current. This represents a practical advantage when implemented in a real MMC as both instantaneous values are readily available to the controller. 


\section{Determination of the Differential Current from Instanta- neous Values. Method 2}

In this subsection, another way of determining the differential current reference from instantaneous values is presented. Assuming high switching frequency, all the SMs in a specific arm will be operated with the same duty cycle. This is equivalent to say that all the SMs in the arm will be activated for some time to represent the activated ones ( $u$ and $l$ ), even when looking into a short timespan. Therefore, the capacitors will be charged/discharged the same because, on average, they will be exposed to the same amount of current. In other words, all $N$ SM capacitors of each arm will evenly share the position of the activated ones within a time interval, as it is represented in Fig. 4. Consequently, the activated SMs present an equivalent (or averaged) capacitance larger than $C$.

The value of the equivalent capacitor can be found from an energy point of view. The energy variation of all the SM capacitors in the upper arm within a time interval $\left[t_{0}, t_{1}\right]$ is:

$$
\Delta \epsilon_{C u}=\epsilon_{C u 1}-\epsilon_{C u 0}=N \frac{1}{2} C\left(v_{C 1}^{2}-v_{C 0}^{2}\right) .
$$

This energy variation has to be the same for the activated SMs assuming that they have an equivalent capacitance $C_{u}^{\prime}$ instead of $C$, as follows:

$$
\Delta \epsilon_{C u}=\epsilon_{C u 1}-\epsilon_{C u 0}=u \frac{1}{2} C_{u}^{\prime}\left(v_{C 1}^{2}-v_{C 0}^{2}\right) .
$$

From (35) and (36), the value of the equivalent SM capacitor is:

$$
C_{u}^{\prime}=\frac{N}{u} C
$$

and similarly for the SM capacitors of the lower arm:

$$
C_{l}^{\prime}=\frac{N}{l} C
$$

Hence, taking into account that there are $u$ and $l$ activated SMs in the upper and lower arms, respectively, the value of the equivalent arm capacitors are:

$$
\begin{gathered}
C_{u_{e q u}}=\frac{C_{u}^{\prime}}{u}=\frac{N}{u^{2}} C \text { and } \\
C_{l_{\text {equ }}}=\frac{C_{l}^{\prime}}{l}=\frac{N}{l^{2}} C .
\end{gathered}
$$

According to (28) and (29), the proper distribution of the arm currents is:

$$
\begin{gathered}
i_{u}=i_{a} \frac{C_{u_{e q u}}}{C_{u_{e q u}}+C_{l_{e q u}}}=i_{a} \frac{l^{2}}{u^{2}+l^{2}} \text { and } \\
i_{l}=i_{a} \frac{C_{l_{e q u}}}{C_{u_{e q u}}+C_{u_{e q u}}}=i_{a} \frac{u^{2}}{u^{2}+l^{2}} .
\end{gathered}
$$

Substituting (30) and (31) into (41) and (42), the arm currents become:

$$
\begin{gathered}
i_{u}=\frac{i_{a}}{2} \frac{\left(1+v_{a m}\right)^{2}}{\left(1+v_{a m}^{2}\right)} \text { and } \\
i_{l}=\frac{i_{a}}{2} \frac{\left(1-v_{a m}\right)^{2}}{\left(1+v_{a m}^{2}\right)} .
\end{gathered}
$$

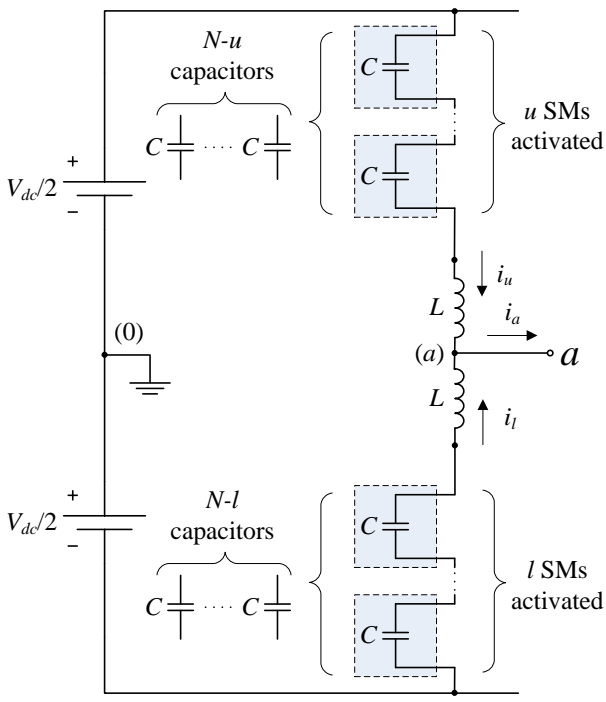

Fig. 4. Phase-leg with equivalent arm capacitances.

Equations (43) and (44) provide the instantaneous references for the arm currents. Considering (4), the differential current is:

$$
i_{d i f f}=\frac{i_{a} v_{a m}}{1+v_{a m}^{2}} .
$$

Equation (45) provides the differential current reference obtained from the instantaneous value of the output current and the modulation signal. If the modulation signal and the output current are sinusoidal, as it was assumed in (11) and (12), and the differential current in (45) is substituted into (13) and (14), the dc power component is not canceled. This means that this method does not provide the proper dc value to the differential current. Nevertheless, this can be compensated by the use of a proportional-integral (PI) controller. On the other hand, the ac component provided by (45) leads to a reduction of the capacitor voltage ripples, as it will be shown in Section VI.

\section{ZERo-SEQUence InJection}

In order to extend the linear operation range of the MMC, a zero-sequence third-order harmonic can be introduced into the modulation signals. Hence, the modulation signal for phase $a$ becomes:

$$
v_{a m}=m_{a} \cos (\omega t)-\frac{m_{a}}{6} \cos (3 \omega t) .
$$

The reference for the differential current with Method 1 is obtained substituting (46) into (34). Assuming that the output current is sinusoidal:

$$
\begin{gathered}
i_{d i f f}=\frac{m_{a} \hat{I_{a}}}{4}[\cos (\varphi)+\cos (2 \omega t+\varphi)] \\
-\frac{m_{a} \hat{I}_{a}}{24}[\cos (2 \omega t-\varphi)+\cos (4 \omega t+\varphi)],
\end{gathered}
$$

and the following harmonic components appear in the power of the arms:

$$
p_{u}, p_{l}=f_{u}(\omega t, 3 \omega t, 4 \omega t, 5 \omega t)
$$


The higher frequency terms are very small and their contribution to the power oscillation is thus little. The fundamental frequency term $(\omega t)$ creates energy fluctuation between the upper and lower arms.

In Method 2, the reference for the differential current is obtained by substituting (46) into (45). Assuming that the output current is sinusoidal:

$$
\begin{gathered}
i_{d i f f}=\frac{m_{a} \hat{I}_{a}}{2} . \\
\frac{\cos (\varphi)+\cos (2 \omega t+\varphi)-\frac{1}{6} \cos (2 \omega t-\varphi)-\frac{1}{6} \cos (4 \omega t+\varphi)}{1+m_{a}\left[\cos (\omega t)-\frac{1}{6} \cos (3 \omega t)\right]} .
\end{gathered}
$$

In this case, many components appear in the power of the arms. Nevertheless, like in Method 1, the most significant ones are those at low frequency.

\section{CAPACITOR Voltage Ripples AND RMS ARM CURRENTS}

The capacitor voltage ripple amplitudes produced in the steady-state operation of the MMC are evaluated in this section. The following conditions and assumptions apply to this analysis:

- The capacitor voltage ripples are obtained from the averaged model of the MMC [18],

- the differential current is imposed to the model for the different cases under analysis,

- because of the nature of the averaged model, ripples at the switching frequency are omitted, and

- a third-harmonic is injected into the modulation signals (46) to achieve maximum linear operation range $\left(m_{a}=[0\right.$, $1.15])$.

According to [18], the capacitor voltages of the upper arm can be represented by:

$$
v_{C u}=\frac{1}{C} \int_{0}^{t} i_{u} \frac{1-v_{a m}}{2} d t+V_{C u 0} .
$$

The capacitor voltages are obtained by imposing $i_{u}$ in (50) for the particular differential current $i_{\text {diff } f}$ under study, i.e. a dc component (16), Method 1 (34), and Method 2 (45). It should be remarked that, in the case of Method 2, the dc component provided by (45) has to be substituted by the dc current given in (16). The capacitor voltage waveform is obtained from (50) over a fundamental period. Then, the peak-to-peak value $\left(\Delta V_{C}\right)$ is determined from the voltage waveform.

The capacitor voltage amplitudes are represented by a normalized magnitude $\left(\Delta V_{C n} / 2\right)$, as follows:

$$
\frac{\Delta V_{C n}}{2}=\frac{\Delta V_{C} / 2}{I_{r m s} / f C}
$$

where $\Delta V_{C}$ is the peak-to-peak ripple, $I_{r m s}$ is the rms value of the output current, $f$ is the fundamental output frequency, and $C$ is the value of the SM capacitor. The arm currents are normalized to the rms output current $\left(I_{r m s}\right)$.

Figs. 5-7 show capacitor voltage ripples and rms arm currents for a dc differential current, Method 1, and Method 2, respectively. As it can be observed, the injection of only a dc component in the differential current produces more voltage ripple amplitudes at large modulation indices than the other methods. On the other hand, it reduces the rms value of the arm current, which eventually leads to lower power losses in the MMC. Method 2 produces lower capacitor voltage ripples than Method 1, with the exception of some small operating areas at very large modulation indices. The rms current values produced by Method 2 are slightly higher than those produced by Method 1, increasing the power losses.

The information provided in Figs. 5-7 regarding normalized capacitor voltage ripple amplitudes is very useful for sizing the MMC capacitors for any given application. For example, let's assume that the converter operates with an output frequency $f=60 \mathrm{~Hz}$, the output current is $I_{r m s}=100 \mathrm{~A}$ with variable power factor, the capacitor voltage reference is $V_{C}^{*}=1000 \mathrm{~V}$, and the modulation index is variable ranging within all the linear interval $\left(m_{a} \in[0,1.15]\right)$. Also, let's assume that the MMC operates with Method 2 and we limit the amplitude of the capacitor voltage ripples to $5 \%$ of $V_{C}^{*}$. From Fig. 7(a), the maximum capacitor voltage ripple is produced at low modulation indices, where the normalized ripple amplitude is:

$$
\frac{\Delta V_{C n}}{2}=0.0563 \text {. }
$$

Therefore, from (51) the minimum capacitor value for this application would be:

$$
C_{\text {min }}=\frac{\Delta V_{C n}}{2} \frac{I_{r m s}}{f \Delta V_{C} / 2}=0.0563 \frac{100}{60 \cdot 50}=0.0019 F .
$$

This value provides an initial estimation of the capacitance based on which the converter can be designed to meet other operating criteria.

The information in Figs. 5-7 can also be used to calculate the capacitor voltage ripples for an specific operating point once the value of the capacitors is defined. If in this example the MMC is operating with a modulation index $m_{a}=0.85$, from Fig. 7(a) and (51) the capacitor voltage ripple amplitude would be:

$$
\frac{\Delta V_{C}}{2}=\frac{\Delta V_{C n}}{2} \frac{I_{r m s}}{f C}=0.0336 \frac{100}{50 \cdot 0.0019}=35.4 \mathrm{~V},
$$

which is less than $50 \mathrm{~V}\left(5 \%\right.$ of $V_{C}^{*}$ ) as we would expected.

\section{BENCHMARKING}

In order to evaluate the circulating current references studied in this paper, the results are benchmarked against those in [26]. The references for the circulating currents in [26] consider two cases; (i) injecting a second harmonic only and (ii) a combination of second and fourth harmonics. The values were obtained off-line by evaluating all the possible amplitudes and angles for the current harmonics injected into the circulating current. Therefore, this direct minimization method guarantees that minimum capacitor voltage ripples are achieved and thus the results can be used as a benchmark for testing the methods studied in this paper.

Fig. 8(a) shows the ratio of capacitor voltage ripples in the case of injecting only a dc circulating current over the results obtained by direct minimization [26] when using an optimal 


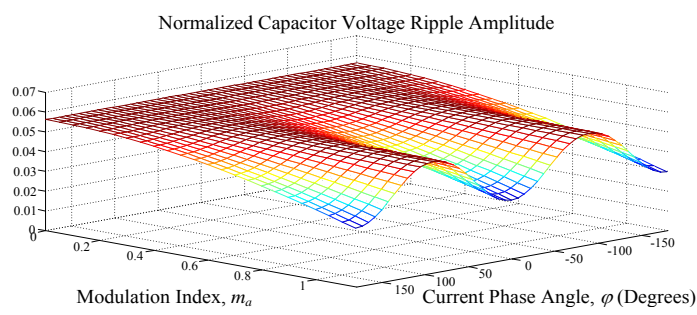

(a)

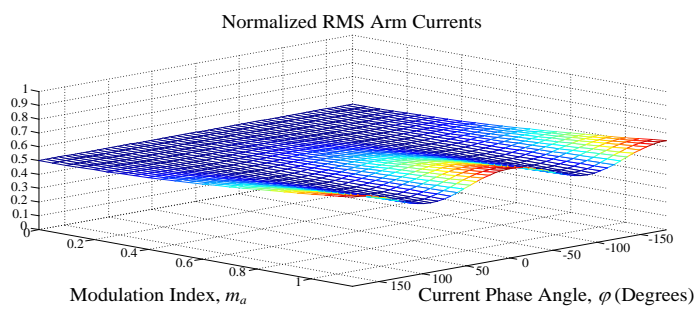

(b)

Fig. 5. A dc differential current: (a) Normalized capacitor voltage ripple amplitude and (b) rms arm current values normalized to the rms output current.

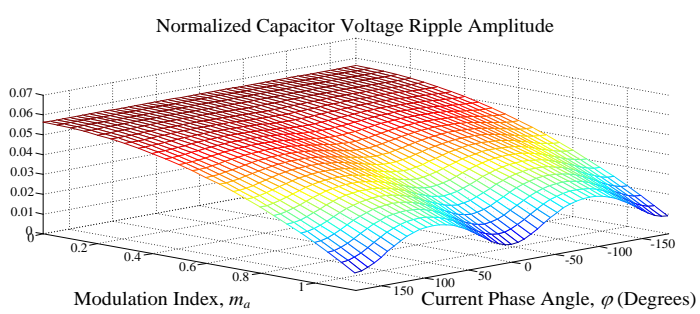

(a)

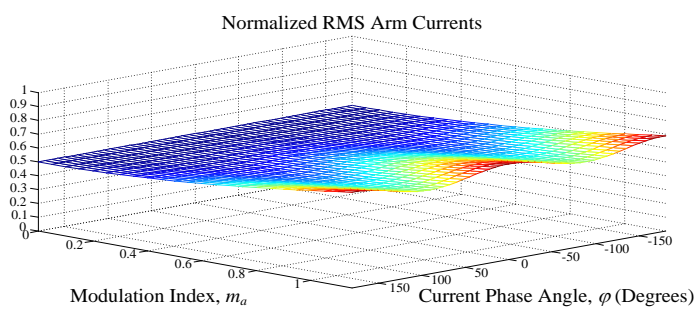

(b)

Fig. 6. Method 1: (a) Normalized capacitor voltage ripple amplitude and (b) rms arm current values normalized to the rms output current.

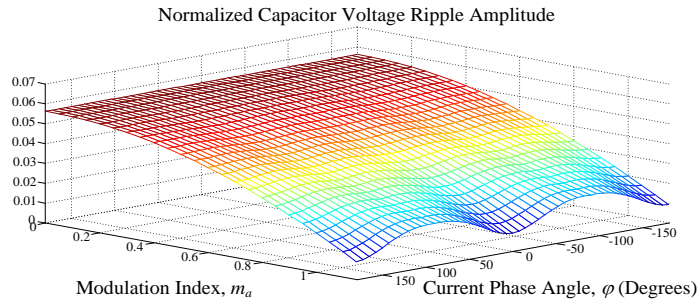

(a)

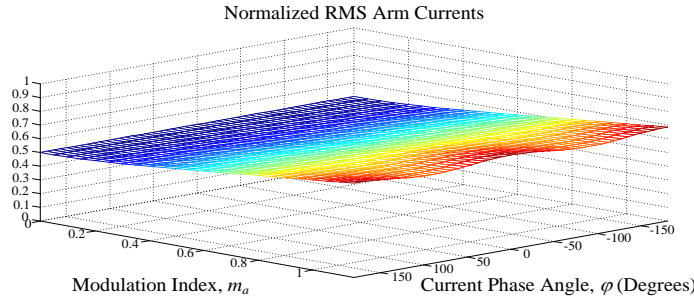

(b)

Fig. 7. Method 2: (a) Normalized capacitor voltage ripple amplitude and (b) rms arm current values normalized to the rms output current.

set of second and fourth harmonics. As it can be appreciated, the ratio of capacitor voltage ripples becomes considerably larger than the unity, especially at large modulation indices (up to about four times larger in the worst case). This means that the capacitor voltage ripples are significantly larger compared to the optimal case. On the other hand, the rms arm current values are lower for all the operating conditions when injecting only a dc current into the circulating current, as it can be seen in Fig. 8(b).

Similar results are shown in Figs. 9 and 10 for the cases of Method 1 and 2. In both cases, the capacitor voltage ripples ratios are relatively close to the unity, which means that Methods 1 and 2 produce capacitor voltage ripples close to the optimal ones. Regarding rms arm currents, Methods 1 and 2 produce lower values than the optimal case [26] for all the operating conditions. Therefore, the direct minimization method in [26] provides optimal results regarding capacitor voltage ripples but at the cost of increasing the rms arm currents and hence the power losses. One can conclude that Methods 1 and 2 produce capacitor voltage ripples close to the minimum ones but with lower rms arm currents. Therefore, they provide a compromise solution between reducing the capacitor voltage ripples and the power losses in the converter. It should be

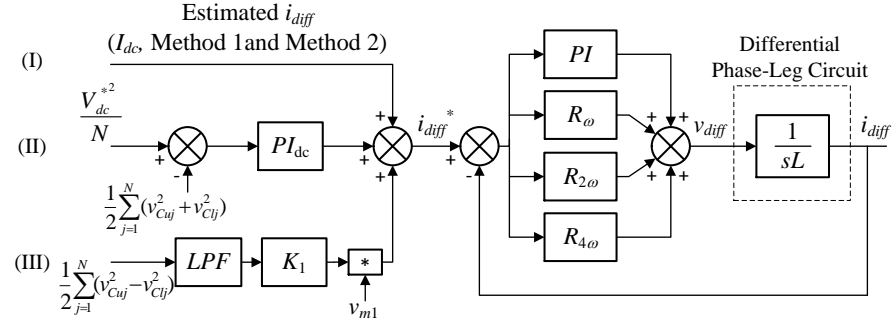

Fig. 11. Scheme of the differential current control.

noted that the implementation of Methods 1 and 2, based on instantaneous values, does not require off-line calculations and is significantly simpler.

\section{DifFERENTIAL CURRENT CONTROL}

As illustrated in Section II, the output and differential currents can be controlled independently. Therefore, any of the well-known current control techniques can be applied to the output current. However, a closed loop current controller for the differential current is necessary to implement the current references given in Section IV. Fig. 11 shows the proposed 


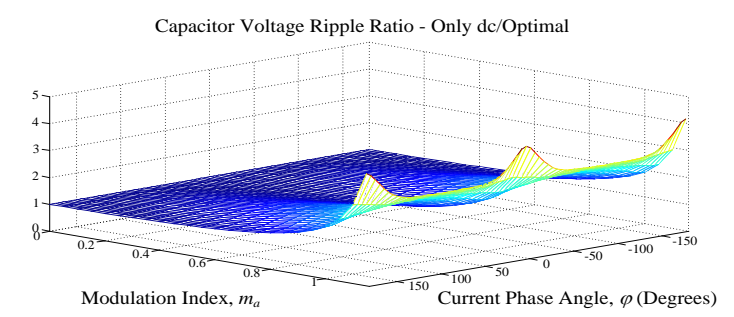

(a)

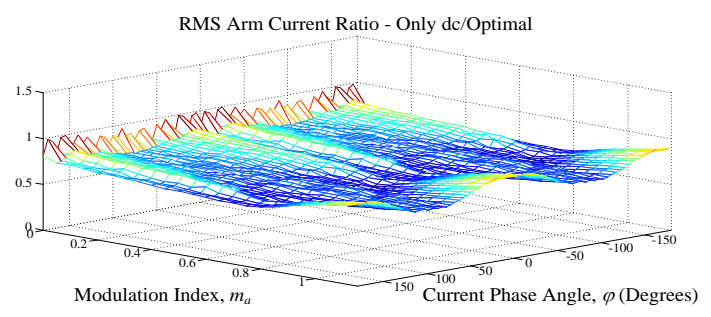

(b)

Fig. 8. A dc component in the circulating current over the optimal case [26]: (a) capacitor voltage ripple ratio and (b) rms arm current ratio.

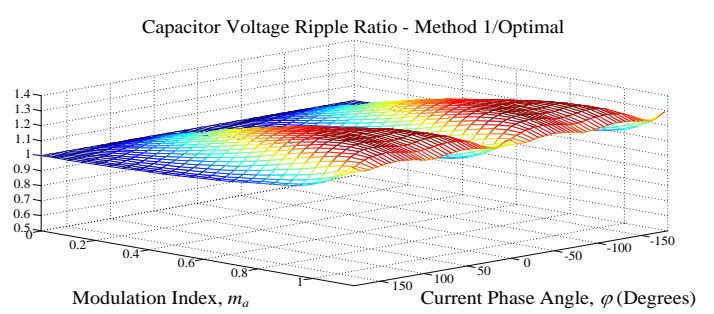

(a)

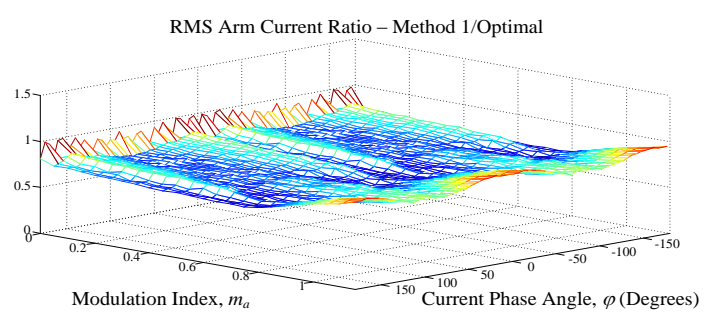

(b)

Fig. 9. Method 1 over the optimal case [26]: (a) capacitor voltage ripple ratio and (b) rms arm current ratio.

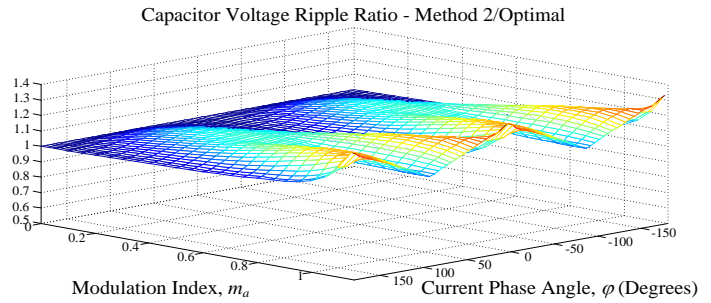

(a)

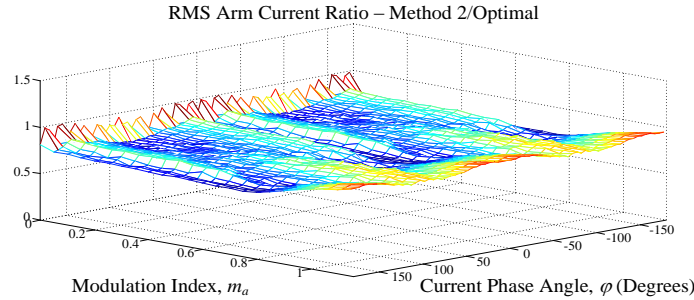

(b)

Fig. 10. Method 2 over the optimal case [26]: (a) capacitor voltage ripple ratio and (b) rms arm current ratio.

differential current control loop where three inputs are required for the definition of the differential current reference $i_{\text {diff }}^{*}$ :

- Component (I): The estimated instantaneous reference current given by (34) or (45). Both current references contain ac and dc components. In the case of Method 1 (34), the estimated value contains the dc current component needed to keep power balance in the phase-leg assuming a lossless MMC. This is therefore a rough estimation of the required dc current component and hence an additional control for the dc current is needed. In the case of Method 2 (45), the reference provided for the dc component is not the proper value and, therefore, the additional control is also needed.

- Component (II): An extra dc current component to maintain the average energy stored in the SM capacitors at its reference value. To determine this dc component, the error between the summation of the quadratic capacitor voltages, which is proportional to the energy stored in the capacitors, and its reference value is calculated. The steady-state error is then driven to zero by a proportionalintegral controller $\left(P I_{d c}\right.$ in Fig. 11).

- Component (III): A fundamental-frequency current component. This current component exchanges energy be- tween the upper and lower arms of each phase-leg; therefore, it assists in maintaining the energy balance between the arms. To achieve optimal performance of the balancing algorithm, this term should be synchronized with the fundamental component of the modulation signal. Its phase is obtained from the modulation signal before a zero sequence is added for the extension of the linear modulation index range $\left(v_{m 1}\right)$. Since there is energy fluctuation between the upper and the lower arms, a low pass filter is required in this loop. The amplitude of this fundamental component of the circulating current is determined by a proportional controller with a gain of $K_{1}$. A proportional controller satisfies the control objective as the control action provided by this controller, when the upper and lower arms are balanced, is zero.

Adding the three above-mentioned components, the differential current reference $\left(i_{\text {diff }}^{*}\right)$ is fed into the current controller shown in Fig. 11. Since the current reference contains a dc term as well harmonics, besides a proportional-integral (PI) controller, a set of resonant $(\mathrm{R})$ controllers tuned at the main frequency components of the current reference, i.e., $\omega, 2 \omega$, and $4 \omega$, can be included. Observe that the harmonic component $3 \omega$ is not tracked although it may appear in the current reference. 


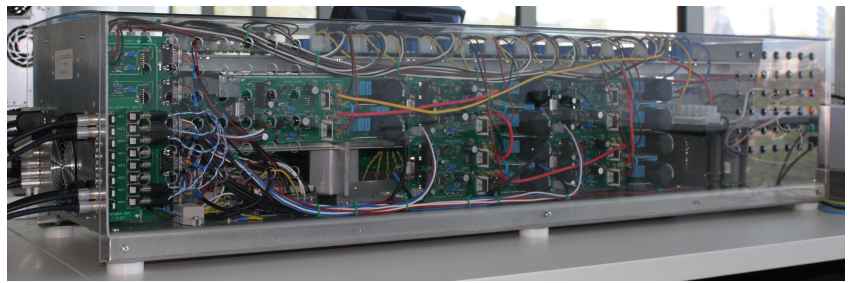

Fig. 12. Experimental setup.

TABLE I

PARAMETERS OF THE EXPERIMENTAL SETUP

\begin{tabular}{l|c}
\hline \multicolumn{1}{c|}{ Parameter } & Value \\
\hline Number of SMs per Phase-Arm, $N$ & 5 \\
\hline SM Capacitors, $C$ & $3.6 \mathrm{mF}$ \\
\hline Phase-Arm Inductors, $L$ & $3.6 \mathrm{mH}$ \\
\hline Load Resistor, $R_{a}$ & $36 \Omega$ \\
\hline Load Inductor, $L_{a}$ & $5 \mathrm{mH}$ \\
\hline Dc-Link Voltage, $V_{d c}$ & $300 \mathrm{~V}$ \\
\hline Dc-Link Capacitors, $C_{d c}$ & $3.3 \mathrm{mF}$ \\
\hline Carrier Frequency, $f_{s}$ & $4 \mathrm{kHz}$ \\
\hline Modulation Index, $m_{a}$ & 0.9 \\
\hline
\end{tabular}

TABLE II

CONTROL PARAMETERS

\begin{tabular}{l|c}
\hline \multicolumn{1}{c|}{ Controller } & Parameters \\
\hline Dc-Link Voltage Control, $P I_{d c}$ & $P=0.011, I=0.07$ \\
\hline Arm Energy Balance Control, $K_{1}$ & $K_{1}=0.021$ \\
\hline Circulating Current Control, $P I \& R_{2 \omega}$ & $P=0.011, I=0, R_{2 \omega}=2.1$ \\
\hline
\end{tabular}

This is because it would produce a current component in the $\mathrm{dc}$ bus that is not canceled by the other phase-legs [25].

\section{EXPERIMENTAL RESUlTS}

The experimental evaluation of the proposed current control and differential current references is performed in a 5-kVA scaled-down, single phase MMC (Fig. 12) with five SMs per arm. A single phase, series connected RL load is connected between the phase terminal and the dc-link mid-point, which is obtained by two series-connected capacitors $C_{d c}$. The control is implemented using a dSPACE dS1103 board. The control structure of Fig. 11 is implemented with a resonant controller tuned at the second harmonic $(2 \omega)$. The system and control parameters are given in Tables I and II.

Three differential current references are provided to the controller, i.e. only a dc component, Method 1 and Method 2. Fig. 13 shows the differential current $\left(i_{d i f f}\right)$ and the output current $\left(i_{a}\right)$ for the three cases under study. As shown in Fig. 13(a), the differential current is almost constant, whereas in Fig. 13(b) a second harmonic is also present. In Fig. 13(c), additional harmonic components in the differential current are present.

Fig. 14 shows the SM capacitor voltages of the upper and lower arms. These were obtained operating with the differential currents shown in Fig. 13. Comparing the results in Fig. 14(a) (only a dc component) with Fig. 14(b) (Method $1)$, the capacitor voltage ripples are reduced significantly

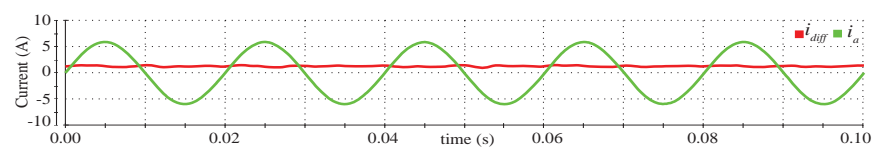

(a)

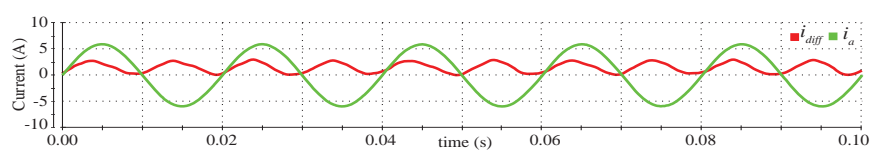

(b)

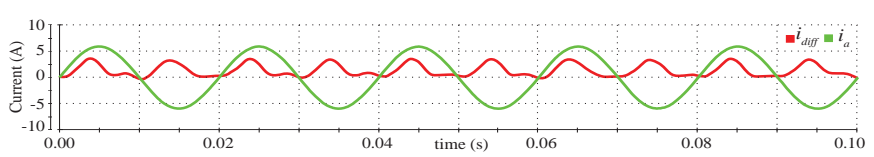

(c)

Fig. 13. Experimental results. Differential current $\left(i_{d i f f}\right)$ and output current $\left(i_{a}\right)$ using the following differential current references: (a) only a dc component, (b) Method 1 and (b) Method 2.

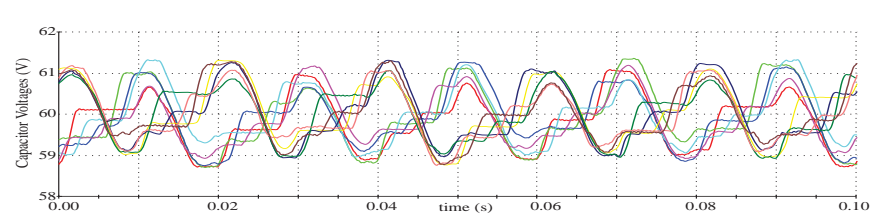

(a)

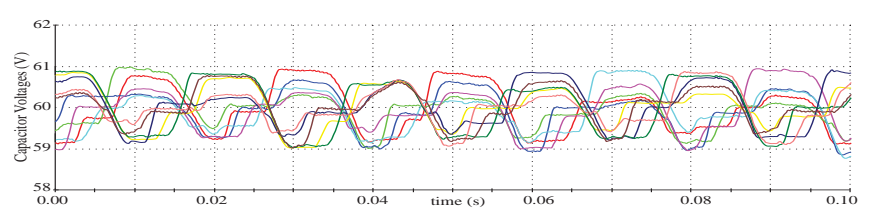

(b)

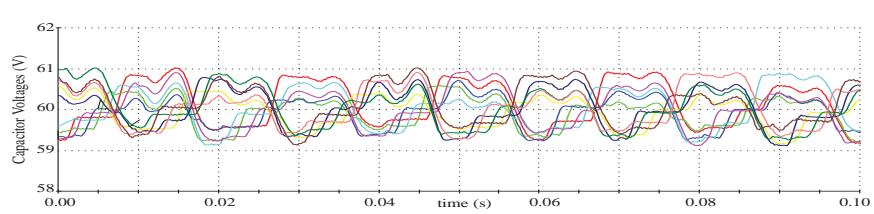

(c)

Fig. 14. Experimental results. SM capacitor voltages using the following differential current references: (a) only a dc component, (b) Method 1 and (b) Method 2.

TABLE III

CAPACITOR VOLTAGE RipPle AMPLITUdES

\begin{tabular}{c|c|c}
\hline $\begin{array}{c}\text { Circulating } \\
\text { Current }\end{array}$ & $\begin{array}{c}\text { Ripple Amplitude } \\
\Delta V_{C} / 2\end{array}$ & $\begin{array}{c}\text { Normalized Amplitude } \\
\Delta V_{C n} / 2\end{array}$ \\
\hline Only dc & $1.30 \mathrm{~V}$ & 0.062 \\
\hline Method 1 & $1.05 \mathrm{~V}$ & 0.050 \\
\hline Method 2 & $0.95 \mathrm{~V}$ & 0.046 \\
\hline
\end{tabular}

when a second harmonic is added to the differential current. Additionally, Fig. 14(c) shows that a further reduction in the capacitor voltage ripple amplitudes can be achieved by using Method 2. Table III summarizes the values of the ripples amplitudes for the three cases. 


\section{Conclusion}

In this paper, a comprehensive analysis of the MMC control has been performed. The instantaneous power in the arms is studied to determine proper differential current references for the phase-arms. Three references have been evaluated and compared in terms of SM capacitor voltage ripples and rms arm currents; i.e. only a dc component, Method 1 and Method 2. It has been shown that Method 2 provides the best results from the point of view of capacitor voltage ripples. Information regarding capacitor voltage ripples has been provided in 3D representations using a normalized magnitude. This information can be used to size the capacitors of the converter for different applications and operating conditions. A controller for the differential current has been proposed that is able to operate with different differential current references. The controller is not only able to control the circulating currents following the references provided, but it also regulates the average capacitor voltages accurately to the reference value and balances the energy between the upper and the lower arms. Experimental results from a low power MMC prototype have shown the good performance of the proposed strategies and the good agreement with the theory.

\section{REFERENCES}

[1] S. Kouro, M. Malinowski, K. Gopakumar, J. Pou, L.G. Franquelo, B. Wu, J. Rodriguez, M.A. Perez, and J.I. Leon, "Recent advances and industrial applications of multilevel converters," IEEE Trans. Ind. Electron., vol. 57, no. 8, pp. 2553-2580, Aug. 2010.

[2] A. Lesnicar and R. Marquardt, "A new modular voltage source inverter topology," in Proc. EPE, Toulouse, France, 2-4 Set. 2003.

[3] A. Lesnicar and R. Marquardt, "An innovative modular multilevel converter topology suitable for a wide power range," in Proc. IEEE Bologna PowerTech Conf., 23-26 Jun. 2003, Bologna, Italy.

[4] M. Hagiwara and H. Akagi, "Control and experiment of pulsewidthmodulated modular multilevel converters," IEEE Trans. Power Electron., vol. 24, no. 7, pp. 1737-1746, Jul. 2009.

[5] M. A. Perez, S. Bernet, J. Rodriguez, S. Kouro, and R. Lizana, "Circuit topologies, modelling, control schemes and applications of modular multilevel converters," to be published IEEE Trans. Power Electron., DOI 10.1109/TPEL.2014.2310127.

[6] S. Debnath, J. Qin, B. Bahrani, M. Saeedifard, and P. Barbosa, "Operation, control, and applications of the modular multilevel converter: A review," to be published IEEE Trans. Power Electron., DOI 10.1109/TPEL.2014.2309937.

[7] H.-J. Knaak, "Multilevel Converters and HVDC/FACTS: a success story," in Proc. EPE, Birmingham, UK, 30 Aug.-1 Set. 2011.

[8] H. Akagi, "Classification, terminology, and application of the modular multilevel cascade converter (MMCC)," IEEE Trans. Power Electron. vol. 26, no. 11, pp. 3119-3130, Nov. 2011.

[9] M. Saeedifard and R. Iravani, "Dynamic performance of a modular multilevel back-to-back HVDC system," IEEE Trans. Power Del., vol. 25, no. 4, pp 2903-2912, Oct. 2010.

[10] H. Mohammadi P. and M. Tavakoli Bina, "A transformerless mediumvoltage STATCOM topology based on extended modular multilevel converters," IEEE Trans. Power Electron., vol. 26, no. 5, pp. 1534-1545, May 2011.

[11] J. Mei, B. Xiao, K. Shen, L. M. Tolbert, and J. Y. Zheng, "Modular multilevel inverter with new modulation method and its application to photovoltaic grid-connected generator," IEEE Trans. Power Electron., vol. 28, no. 11, pp. 5063-5073, Nov. 2013.

[12] S. Debnath and M. Saeedifard, "A new hybrid modular multilevel converter for grid connection of large wind turbines," IEEE Trans. Sustain. Energy, vol. 4, no. 4, pp. 1051-1064, Oct. 2013.

[13] A. J. Korn, M. Winkelnkemper, and P. Steimer, "Low output frequency operation of the modular multi-level converter," in Proc. IEEE ECCE, Atlanta, GA, USA, 12-16 Sep. 2010, pp. 3993-3997.
[14] J. Kolb, F. Kammerer, and M. Braun, "Straight forward vector control of the modular multilevel converter for feeding three-phase machines over their complete frequency range," in Proc. IEEE IECON, Melbourne, Australia, 7-10 Nov. 2011, pp. 1596-1601.

[15] K. Wang, Y. Li, Z. Zheng, and L. Xu, "Voltage balancing and fluctuationsuppression methods of floating capacitors in a new modular multilevel converter," IEEE Trans. Ind. Electron., vol. 60, pp. 1943-1954, May 2013.

[16] M. Saeedifard, R. Iravani, and J. Pou, "Analysis and control of DCcapacitor-voltage-drift phenomenon of a passive front-end five-level converter," IEEE Trans. Ind. Electron., vol. 54, pp. 3255-3266, Dec. 2007.

[17] J. Qin and M. Saeedifard, "Reduced switching-frequency voltagebalancing strategies for modular multilevel HVDC converters," IEEE Trans. Power Del., vol. 28, no. 4, pp. 2403-2410, Oct. 2013.

[18] S. Ceballos, J. Pou, S. Choi, M. Saeedifard, and V. G. Agelidis, "Analysis of voltage balancing limits in modular multilevel converters," in Proc. IEEE IECON, 7-10 Nov. 2011, Melbourne, Australia, pp. 4397-4402.

[19] L. Harnefors, A. Antonopoulos, S. Norrga, L. Ängquist, and Hans-Peter Nee, "Dynamic analysis of modular multilevel converters," IEEE Trans. Ind. Electron., vol. 60, no. 7, pp. 2526-2537, Jul. 2013.

[20] M. Vasiladiotis, N. Cherix, and A. Rufer, "Accurate capacitor voltage ripple estimation and current control considerations for grid-connected modular multilevel converters," IEEE Trans. Power Electron., vol. 9, no. 9, pp. 4568-4579, Sep. 2014.

[21] Q. Tu, Z. Xu, and L. Xu, "Reduced switching-frequency modulation and circulating current suppression for modular multilevel converters," IEEE Trans. Power Del., vol. 26, no. 3, pp. 2009-2017, Jul. 2011.

[22] R. Darus, J. Pou, G. Konstantinou, S. Ceballos, and V. G. Agelidis, "Circulating current control and evaluation of carrier dispositions in modular multilevel converters," in Proc. IEEE ECCE Asia, Melbourne, Australia, 3-6 Jun. 2013, pp. 332-338

[23] K. Ilves, S. Norrga, L. Harnefors, and H.-P. Nee,"On energy storage requirements in modular multilevel converters," in IEEE Trans. Power Electron., vol. 29, no. 1, pp. 77-88, Jan. 2014.

[24] R. Picas, J. Pou, S. Ceballos, V. G. Agelidis, and M. Saeedifard, "Minimization of the capacitor voltage fluctuations of a modular multilevel converter by circulating current control," in Proc. IEEE IECON, Montreal, Canada, 25-28 Oct. 2012, pp. 4985-4991.

[25] S. P. Engel and R. W. De Doncker, "Control of the modular multi-level converter for minimized cell capacitance," in Proc. EPE, Birmingham, UK, 30 Aug.-1 Set. 2011.

[26] R. Picas, J. Pou, S. Ceballos, J. Zaragoza, G. Konstantinou, and V. G. Agelidis, "Optimal injection of harmonics in circulating currents of modular multilevel converters for capacitor voltage ripple minimization," in Proc. IEEE ECCE Asia, Melbourne, Australia, 3-6 Jun. 2013, pp. 318324

[27] M. Winkelnkemper, A. Korn, and P. Steimer, "A modular direct converter for transformerless rail interties," in IEEE ISIE, Bari, Italy, 4-7 Jul. 2010 , pp. 562-567.

[28] J. Pou, S. Ceballos, G. Konstantinou, G. J. Capella, and V. G. Agelidis, "Control strategy to balance operation of parallel connected legs of modular multilevel converters," in Proc. IEEE ISIE, Taiwan, 28-31 May 2013.

[29] E. Solas, G. Abad, J. Andoni Barrena, S. Aurtenetxea, A. Carcar, and L. Zajac, "Modular multilevel converter with different submodule concepts-Part I: capacitor voltage balancing method," IEEE Trans. Ind. Electron., vol. 60, no. 10, pp. 4525-4535, Oct. 2013.

[30] E. Solas, G. Abad, J. Andoni Barrena, S. Aurtenetxea, A. Carcar, and L. Zajac, "Modular multilevel converter with different submodule concepts-Part II: experimental validation and comparison for HVDC application," IEEE Trans. Ind. Electron., vol. 60, no. 10, pp. 4536-4545, Oct. 2013.

[31] Z. Li, P. Wang, H. Zhu, Z. Chu, and Y. Li, "An improved pulse width modulation method for chopper-cell-based modular multileve converters," IEEE Trans. Power Electron., vol. 27, no. 8, pp. 3472-3481, Aug. 2012. 


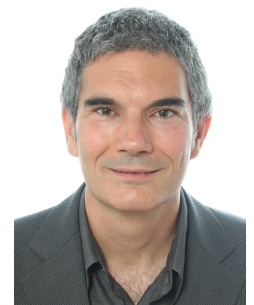

Josep Pou (S'97-M'03-SM'13) received the B.S. M.S., and Ph.D. degrees in electrical engineering from the Technical University of Catalonia (UPC), Catalonia, Spain, in 1989, 1996, and 2002, respectively.

In 1990, he joined the faculty of UPC as an Assistant Professor, becoming an Associate Professor in 1993. Since February 2013, he has been a Professor with the University of New South Wales (UNSW), Sydney, Australia, on leave from UPC, where he keeps a permanent position. In 2001 and 2006, he was a Researcher with the Center for Power Electronics Systems, Virginia Polytechnic Institute and State University, Blacksburg, VA, USA. In 2012, he was a Researcher with the Australian Energy Research Institute, School of Electrical Engineering and Telecommunications, UNSW. He is the author of more than 170 published technical papers, and has been involved in severa industrial projects and educational programs in the fields of power electronics and systems. His research interests include multilevel converters, renewable energy generation, and high-voltage dc transmission systems.

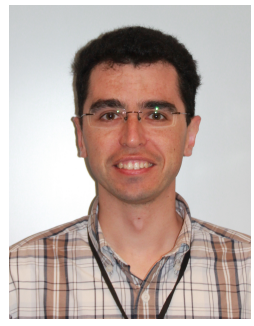

Salvador Ceballos received the M.S. degree in physics from the University of Cantabria, Santander Spain, in 2001, and the M.S. and Ph.D. degrees in electronic engineering from the University of the Basque Country, Bilbao, Spain, in 2002 and 2008 respectively.

Since 2002 he has been with Tecnalia Research and Innovation, where he is currently a researcher in the Energy and Environmental Division. From May 2008 to May 2009 he was a visiting researcher at the Hydraulic and Maritime Research Centre (HMRC) University College Cork (UCC), Cork, Ireland. He has authored more than 80 published technical papers. His research interests include multilevel converters, fault-tolerant power electronic topologies, and renewable energy systems.

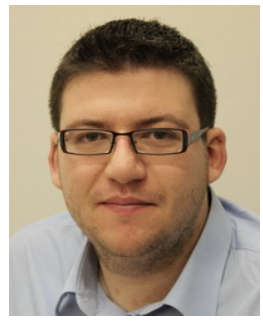

Georgios Konstantinou (S'08-M'13) received his B.Eng. in Electrical and Computer Engineering from Aristotle University of Thessaloniki, Thessaloniki, Greece in 2007 and his Ph.D. degree in Electrical Engineering from UNSW Australia, Sydney, Australia in 2012.

He is currently a Senior Research Associate with the Australian Energy Research Institute (AERI) and the School of Electrical Engineering and Telecommunications, UNSW Australia. His research interests include hybrid and modular multilevel converters, pulse-width modulation and selective harmonic elimination techniques for power electronics. He is an Associate Editor of IET Power Electronics.

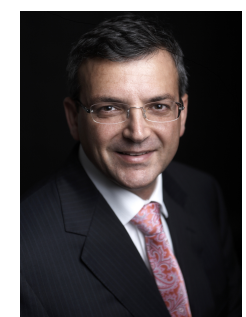

Vassilios G. Agelidis (SM'00) was born in Serres, Greece. He received the B.Eng. degree in electrical engineering from the Democritus University of Thrace, Thrace, Greece, in 1988; the M.S. degree in applied science from Concordia University, Montreal, QC, Canada, in 1992; and the Ph.D. degree in electrical engineering from Curtin University, Perth, Australia, in 1997.

$\mathrm{He}$ has worked with Curtin University (1993-1999); the University of Glasgow, Glasgow, U.K. (2000-2004); Murdoch University, Perth, Australia (2005-2006); and the University of Sydney, Sydney, Australia (2007-2010). He is currently the Director of the Australian Energy Research Institute, School of Electrical Engineering and Telecommunications, University of New South Wales, Sydney.

Dr. Agelidis was the recipient of the Advanced Research Fellowship from the U.K.'s Engineering and Physical Sciences Research Council in 2004 $\mathrm{He}$ was the Vice President for Operations with the IEEE Power Electronics Society (PELS) from 2006 to 2007. He was an AdCom Member of IEEE PELS from 2007 to 2009 and the Technical Chair of the 39th IEEE Power Electronics Specialists Conference in 2008 held in Rhodes, Greece.

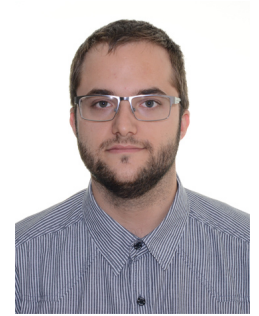

Ricard Picas (S'12) received the B.S. degree in electronic engineering and the M.S. degree in automatics and industrial electronics engineering from the Technical University of Catalonia (UPC), Terrassa, Spain, in 2010 and 2012, respectively. He is currently pursuing the Ph.D. degree at the UPC.

Since 2012 he is working with the Terrassa Industrial Electronics Group (TIEG) at UPC in Terrassa as a Ph.D. student. His main research interests include multilevel converters and HVDC systems.

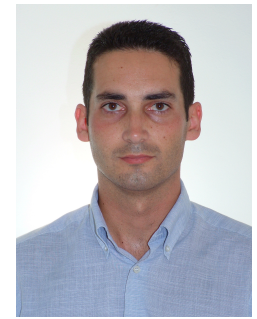

Jordi Zaragoza (S'08-M'12) received the B.S degree in electronic engineering, the M.S. degree in automatic and electronic industrial engineering and $\mathrm{Ph} . \mathrm{D}$. degree from the Technical University of Catalonia (UPC), Catalonia, Spain, in 2001, 2004, and 2011 respectively.

In 2003, he joined the faculty of UPC as an Assistant Professor, where he became an Associate Professor in 2012. From September 2006 to September 2007 he was a researcher at the Energy Unit of ROBOTIKER-TECNALIA Technologic Corporation, Basque Country, Spain. $\mathrm{He}$ is the author of more than 50 published technical papers and has been involved in several projects in the fields of power electronics and systems. His research interests include modeling and control of power converters, multilevel converters, wind energy, power quality and HVDC transmission systems. 